\title{
Reducing short-stay hospital admissions by ruling out non-ST elevation myocardial infarction and estimating coronary artery disease likelihood on an emergency department observation ward
}

Martin Wiese

\begin{abstract}
Chest pain is an important presentation in adult patients attending emergency departments (ED). The process of ruling out an acute coronary syndrome (ACS) conventionally requires a short in-patient stay. This places a significant burden on healthcare systems. Recent developments have encouraged us to explore the role of an ED observation ward in the management of these patients.

We designed and implemented two proformas ('flowformas'). The first provides integrated guidance and documentation for the management of chest pain in the ED. In patients determined to be at low risk of short-term adverse outcomes the ACS rule-out process is now completed on the ED observation ward rather than on the cardio-respiratory admission ward. The second proforma is used before discharge to determine the likelihood of underlying coronary artery disease (CAD), thereby allowing risk-based follow-up arrangements to be made. We collected data on all patients admitted to EDU on the NSTEMI rule-out pathway over a 12-month period.
\end{abstract}

Between Feb 2012 and Feb 2013, 816 patients fulfilling the criteria were admitted on the pathway. 67 patients (8\%) required admission due to ACS. 15 patients were admitted on two, and one on three occasions.

In conclusion, it is possible to deliver ACS rule-out on an emergency observation ward. This reduces healthcare costs and shortens hospital stay.

\section{Problem}

An ageing population, recent changes in healthcare economics and a harsh current financial climate are all putting severe pressure on the availability of acute hospital beds, often causing considerable overcrowding in emergency departments. This has prompted an interest in ambulatory care and observation medicine as alternatives to avoidable short-term hospital admissions.

Specialist services in our Trust are distributed across three sites. ED patients requiring acute admission have to be transported to the cardio-respiratory admission unit, situated at one of the other hospitals. This is not only costly but can be irritating to patients who often find themselves being discharged not long after arrival at the other site.

Appropriate care for patients with chest pain of recent onset does not end after ACS has been ruled out but requires careful consideration of the likelihood of undiagnosed coronary artery disease (CAD) in order to determine the correct follow-up arrangements. This falls out of the expertise of most emergency physicians trained in the UK.

\section{Background}

The mortality rate from ischaemic heart disease for the population of Leicester is amongst the highest in England. [1] Contributing factors include high levels of social deprivation and alcohol misuse, and a large South East Asian population. The ED team wanted to help improve health outcomes in this area.

Patients with a suspected acute coronary syndrome (ACS) or nonST elevation myocardial infarction (NSTEMI) but without obvious early evidence of cardiac ischaemia have until recently required hospital admission for determination of cardiac troponin blood levels 12 hours from the onset of their most recent episode chest pain.

In the last few years, advances in healthcare technology through the advent of highly sensitive troponin assays have made it possible to shorten the ACS rule-out time dramatically. [2,3] The 2011 'European Society of Cardiology (ESC) guidelines for the management of acute coronary syndromes in patients presenting without persistent ST-segment elevation' support 'a rapid rule-out protocol ( 0 and 3 hours) when highly sensitive troponin tests are available'. [4]

The 'Global Registry of Acute Coronary Events' (GRACE) score, advocated in the 2010 NICE clinical guideline 95 on 'chest pain of recent onset' ('CG95') [5] allows identification of a subpopulation of patients with acute chest pain who are at low risk of adverse outcomes while in hospital and during the following six months.

The same NICE guideline also outlined a strategy for clinical 
estimation of CAD likelihood based on typicality of symptoms, age, sex, and the presence or absence of all or none of three risk factors (smoking, diabetes and hyperlipidaemia).

Ambulatory models of care for ED patients with chest pain are being actively explored in several countries, including the US. [6]

\section{Baseline measurement}

A retrospective chart review of all adult patients attending our ED with chest pain in June 2011 identified 223 patients requiring admission to rule out ACS, or around seven per day.

Based on the new model of care, we estimated that around five patients per day might be suitable for NSTEMI rule-out completion on the ED observation ward (in our Trust called 'Emergency Decision Unit', or EDU).

\section{Design}

First, we implemented a rapid NSTEMI rule-out protocol as recommended by the ESC, thereby utilising the highly sensitive troponin assay already in use throughout the Trust to its best advantage.

Next, we designed two proformas containing all the documentation and guidance elements (termed 'flowformas') required by ED clinicians to manage patients with acute chest pain of suspected cardiac origin who are deemed to be 'low-risk' from arrival to discharge from EDU. The concept and style of those flowformas follows the format of a well established suite of guidance and documentation tools in our department.

In our new model of care, patients are considered 'low-risk' if their risk of death within 6 months as determined by GRACE score is no greater than $3 \%$, if they are (at least hypothetically) fit enough to undergo an exercise ECG, and if they have no established CAD with suspected crescendo angina.

Although NICE CG95 makes no recommendation about the specific setting in which CAD likelihood assessment should take place we felt that this would add significant value by allowing risk-based determination of the appropriate follow-up arrangements.

The CAD likelihood estimation table propagated by the NICE guideline shows the percentage of people estimated to have coronary artery disease according to typicality of symptoms, age, sex and the absence (i.e. low) or presence of all three (i.e. high) risk factors. Following correspondence with the chair of the NICE guideline development group, we adapted the table to also show the percentage of people with just one or two risk factors estimated to have $C A D$

Patients are informed about their estimated CAD likelihood before discharge and given a copy of their risk assessment and ECG. Those with an estimated likelihood of $>60 \%$ are referred directly for an exercise ECG. Patients with a likelihood of $<61 \%$ are referred back to their GP, with specific recommendations for further care.

\section{Strategy}

The project was initiated at a Trust ambulatory care board meetings in summer 2011, which brought together colleagues from acute and respiratory medicine, cardiologists and cardiac nurse practitioners (CNPs).

\section{PDSA Cycle 1}

We designed a first draft of the NSTEMI (non-ST elevation myocardial infarction) rule-in/out proforma following agreement about the 'low-risk' criteria with the nominated consultant cardiologist. We initially specified a six-hour NSTEMI rule-out protocol. The first draft of the CAD likelihood assessment proforma assumed that the ED would be able to refer suitable patients directly for CAD diagnostic testing, but this model of care had to be put on hold pending appropriate expansion of testing capacity within the Trust.

PDSA Cycle 2

The early drafts contained a lot of detail about the management of diagnosed NSTEMI, including the anti-platelet and anti-thrombin strategies recommended NICE CG95. These had to be removed due to lack of consensus within cardiology.

PDSA Cycle 3

Following publication of the ESC NSTEMI management guidelines, we agreed to shorten the rule-out protocol to three hours (from first troponin measurement). The protocol was launched Trust-wide with a presentation at the medical grand round, followed by a news bulletin on the hospital intranet.

\section{PDSA Cycle 4}

After encouraging tests of the proformas in selected patients by the CNPs we embarked on a two-month pilot from February 2012. During the pilot, ED staff were supported by a program of educational interventions including brief educational interventions and material for self-directed learning, detailing use of the proformas in a range of scenarios. One area of persistent difficulty seemed to be the correct operation of the online GRACE calculator; this was addressed by placing pictorial user instructions on the back of the NSTEMI rule-in/out proforma.

\section{PDSA Cycle 5}

In response to feedback from users the recommendations to GPs regarding further care were reformatted into a preprinted letter with tick boxes allowing convenient selection of the appropriate options on the back of the CAD likelihood assessment proforma.

PDSA Cycle 6

The 'low-risk chest pain pathway' was fully implemented at the end 
of the pilot, and, following the appropriate coding change notice, the ED has been able to collect the same-day emergency care best practice tariff for patients with chest pain. Post-measurement was carried out 12 months after rolling out the new care model.

\section{Results}

Between Feb 2012 and Feb 2013, 816 patients fulfilling the low-risk criteria were admitted on the pathway to EDU.

67 patients (8\%) required admission due to ACS.

15 patients were admitted on two, and one on three occasions.

\section{Lessons and limitations}

During the project we came to appreciate the value of a champion who ensured that implementation did not stall due to the complex decision-making processes and sometimes obscure lines of command in a large organisation such as ours.

The success of the project has highlighted the skills and expertise of our emergency physicians 'beyond the first four hours". It has helped pave the way for additional ambulatory care EDU pathways (four so far, and counting), and we are confident that our vision of being able to provide CAD diagnostic testing from the ED will soon become a reality. It is nevertheless important to stress that sustainability is dependent on regular training for all new starters as well as constant vigilance against protocol violations.

Our figures suggest that we are recruiting less patients for the pathway than anticipated (on average two rather than five patients per day). Further work is required to explore the reasons for this.

Further work is also needed to quantify any cost savings made through implementation of the pathway, but the reduced need for inter-hospital ambulance transfer and assessment by a second (inhospital) team make substantial savings likely.

We did not seek to evaluate the safety of the three-hour NSTEMI rule-out protocol, since this has previously been established. [2-4]

\section{Conclusion}

In conclusion, implementation of an ED observation unit care pathway to rule out NSTEMI in low-risk patients is feasible. This helps avoid short-stay hospital admissions and is likely to reduce healthcare costs.

\section{References}

1. Hughes D. Heart disease deaths highest in north-west England. BBC News Health. 7 July 2011. http://www.bbc.co.uk/news/health-14048969 (accessed 15 April 2013).
2. Keller T, Zeller T, Peetz D et al. Sensitive troponin I assay in early diagnosis of acute myocardial infarction. NEJM 2009;361:868-77.

3. Reichlin T, Hochholzer W, Bassetti S et al. Early diagnosis of myocardial infarction with sensitive cardiac troponin assays. NEJM 2009;361:858-67.

4. Hamm CW, Bassand JP, Agewall S et al. ESC guidelines for the management of acute coronary syndromes in patients presenting without persistent ST-segment elevation. The Task Force for the management of acute coronary syndromes (ACS) in patients presenting without persistent ST-segment elevation of the European Society of Cardiology (ESC). Eur Heart J 2011;32:2999-3054

5. National Institute for Health and Clinical Excellence. Chest pain of recent onset: Assessment and diagnosis of recent onset chest pain or discomfort of suspected cardiac origin. NICE clinical guideline 95. London: NICE; 2010.

6. Hess EP, Knoedler MA, Shah ND et al. The chest pain choice decision aid: A randomised trial. Circ Cardiovasc Qual Outcomes 2012;5:251-9.

\section{Declaration of interests}

Nothing to declare

\section{Acknowledgements}

Thanks to Amanda Cooper, Glen Sibbick and the whole team of CNPs for carrying out the baseline survey. Huge thanks is also required to Dr. Mandar Marathe for assisting implementation of the pilot, consultant cardiologist Dr. lan Hudson for championing the pathway, consultant chemical pathologist Dr. Webster Madira for his counsel on using high-sensitivity troponins, and the ED nursing and medical staff who have willingly implemented these changes so effectively despite working in an extremely challenging environment. 